\title{
Blockchain al servicio del periodismo de calidad. El caso Civil
}

\section{Blockchain at the service of quality journalism: the Civil case}

\author{
Marçal Sintes-Olivella; Enric Xicoy-Comas; Elena Yeste-Piquer
}

Cómo citar este artículo:

Sintes-Olivella, Marçal; Xicoy-Comas, Enric; Yeste-Piquer, Elena (2020). “Blockchain al servicio del periodismo de calidad. El caso Civil". Profesional de la información, v. 29, n. 5, e290522.

https://doi.org/10.3145/epi.2020.sep.22

Artículo recibido el 22-03-2020

Aceptación definitiva: 08-05-2020
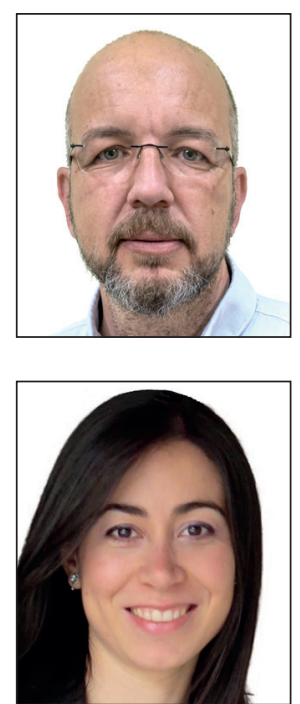

Marçal Sintes-Olivella

https://orcid.org/0000-0001-7521-3033

Universitat Ramon Llull

Facultat de Comunicació i Relacions

Internacionals Blanquerna

Plaça Joan Coromines, $\mathrm{s} / \mathrm{n}$.

08001 Barcelona, España

marcalso@blanquerna.url.edu

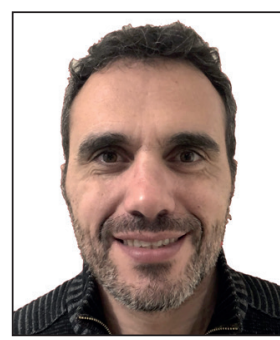

Enric Xicoy-Comas $\bowtie$

https://orcid.org/0000-0003-2638-8456

Universitat Ramon LLull

Facultat de Comunicació $i$ Relacions

Internacionals Blanquerna

Plaça Joan Coromines, $\mathrm{s} / \mathrm{n}$.

08001 Barcelona, España

enricxc@blanquerna.url.edu

\section{Resumen}

Nos encontramos en una era marcada por la desinformación, las fake news y la crisis del periodismo. A la crisis económica, que ha provocado la desaparición de puestos de trabajo y numerosos medios de comunicación convencionales, especialmente en el sector de la prensa impresa, hay que sumar una intensa crisis de confianza. Entre las opciones para intentar salir de esta situación, varios teóricos de la comunicación y algunas empresas consideran que la tecnología blockchain ofrece nuevas oportunidades al periodismo de calidad. La cadena de bloques tiene su origen en la moneda electrónica, pero cada vez está siendo más utilizada en la comunicación digital y el periodismo en particular. Este artículo está dedicado al caso de Civil, la primera plataforma de blockchain para periodismo, que alojaba a decenas de medios de comunicación que habían adquirido el compromiso de respetar las normas éticas impuestas por la organización. El presente estudio de caso centra su atención en los siguientes aspectos: la misión y objetivos del proyecto Civil, su origen e historia, la estructura y el sistema de gobernanza de la organización, la función de la criptomoneda propia y los principios éticos y normas profesionales que rigen la llamada Comunidad Civil. Para estudiar Civil no sólo nos hemos fijado en los documentos de su web y en la bibliografía existente, sino que llevamos a cabo entrevistas a miembros del proyecto, algunas en su sede de Nueva York. Asimismo, entrevistamos a la responsable del primer medio de comunicación que adoptó la tecnología blockchain a través de esta plataforma.

\section{Palabras clave}

Periodismo; Medios de comunicación; Blockchain; Criptomoneda; Ética periodística; Periodismo de calidad; Tecnologías; Innovación; Constitución Civil; Civil.

\section{Abstract}

We are in an era marked by misinformation, fake news, and a journalistic crisis. To the economic crisis that has resulted in the disappearance of jobs and numerous conventional media, especially in the printed press sector, one must add 
an intense crisis of trust. Among the options to escape from this situation, several communication theorists and some companies believe that blockchain technology offers new opportunities for high-quality journalism. Blockchain has its origin in electronic currencies, but it is increasingly being used in digital communication and journalism. This article is dedicated to the case of Civil, the first blockchain platform for journalism, which hosted tens of media, all accepting the commitment to respect the ethical standards imposed by the organization. This case study focuses on the following aspects: the mission and objectives of the Civil project, its origin and history, the structure and governance system of the organization, the role of the cryptocurrency itself, and the ethical principles and professional standards that govern the so-called Civil Community. In order to study Civil, we not only looked at the documents on its website and existing literature, but also carried out interviews with project members, some of them at its headquarters in New York. Likewise, we interviewed the person in charge of the first media that adopted blockchain technology through this platform.

\section{Keywords}

Journalism; Media; Blockchain; Cryptocurrency; Journalistic ethics; Quality journalism; Technologies; Innovation; Civil constitution; Civil.

\section{Introducción}

La revolución tecnológica relacionada con internet y las redes sociales se encuentra en la base de la crisis del periodismo y de los medios de comunicación que estamos viviendo, y que ha supuesto el fin de la posición de privilegio que tradicionalmente han ostentado estos últimos (Anderson; Bell; Shirky, 2015; Blumler; Kavanagh, 1999; Jarvis, 2014; Sunstein, 2002). Los cambios tecnológicos contribuyen a poner en cuestión el modelo convencional de negocio periodístico, que ha sufrido una importante caída en los ingresos. Además, los medios de comunicación han visto erosionada la confianza del público en el periodismo. Esta pérdida de confianza la refleja, por ejemplo, el Edelman Trust Barometer de 2020, donde podemos leer que un $57 \%$ de la población considera que

“los medios que consumo están contaminados con información no confiable” (Edelman, 2020, p. 16).

En el mismo informe se apunta que en España y Estados Unidos la confianza en los medios no llega al $50 \%$ : un $42 \%$ y $48 \%$ respectivamente (Edelman, 2020, p. 42).

La crisis que padece el periodismo escrito desde principios del siglo XXI-y que se vio agravada por el desplome de la economía de 2008- ha obligado a los medios a un replanteamiento global del modelo de negocio. Se trata de una crisis que sobre todo ha afectado el sector del papel. El informe Newspapers fact sheet, del Pew Research Center (2019), señala que en 2007 se vendían 50,7 millones de ejemplares de diarios entre semana en EUA, cifra que fue disminuyendo hasta 28,5 millones en 2018. Lo mismo ocurre con los puestos de trabajo en el sector de la prensa, que pasaron de 73.810 en 2007 a 37.900 en 2018.

Mientras que el sector de los medios de comunicación permaneció estable durante la segunda mitad del siglo XX, no se puede decir lo mismo de lo que llevamos del XXI. Muchas compañías han optado por buscar nuevas fuentes de ingresos para hacer frente a la importante caída de venta de periódicos, caída que se produjo también en la inversión publicitaria.

La mayoría de las empresas más importantes del sector están todavía estudiando cuál es la mejor o las mejores vías para hacer frente a los retos a los que se enfrenta el periodismo en el nuevo entorno digital, con un doble objetivo:

- recuperar la confianza del público;

- revertir el constante descenso en las cifras de negocio.

Ambos objetivos están íntimamente relacionados.

Una de las herramientas que pueden contribuir a la consecución de los dos objetivos arriba señalados es blockchain, una tecnología digital unida al nacimiento de la primera criptomoneda, el bitcoin.

Friedman lo explica en su libro Gracias por llegar tarde:

“En 2007, Satoshi Nakamoto -nombre utilizado por una persona o personas desconocidas- empezó a trabajar en una moneda y sistema de pago digital llamado bitcoin. El 31 de octubre de 2008, Nakamoto comunicó el concepto en un artículo de investigación titulado 'Bitcoin: A peer-to-peer electronic cash system'”' (Friedman, 2018, p. 33).

Bitcoin fue el primer ejemplo de aplicación de blockchain, en este caso para crear una criptomoneda, que actualmente copa casi la mitad del mercado. Hoy día no es la única criptomoneda, pues se contabilizan más de mil tipos. Ether, moneda creada en 2015 y que opera a través de la plataforma de blockchain Ethereum, es otra moneda virtual de referencia. Apunta Boulosa que

"las transacciones de ether son igualmente más rápidas que las de bitcoin, pues Ethereum confirma transacciones en segundos, mientras que bitcoin requiere minutos en el mejor de los casos" (Boulosa, 2019, p. 132).

No fue hasta la segunda mitad de los años 10 cuando empezó a difundirse la utilidad de blockchain en campos distintos del estrictamente monetario. 
La traducción de blockchain al español es "cadena de bloques". Cada bloque es un paquete de información que distintos usuarios -nodos-, verifican y almacenan mediante un sofisticado sistema de encriptación, lo que asegura que una vez se han incorporado a la cadena, los datos no son manipulables retroactivamente. EI hecho de que los bloques estén vinculados entre sí les dota de trazabilidad. Entre la información guardada de forma descentralizada -distribuida- se halla la autoría, que, sin embargo, el sistema permite anonimizar. Los paquetes de información archivados pueden ser publicaciones concretas: reportajes, entrevistas, informes, fotografías, vídeos, etcétera.

Sobre las posibilidades que ofrece la tecnología blockchain al periodismo, resulta especialmente interesante la aportación de Al-Saqaf y Picha-Edwardsson (2019), quienes señalan aquellas áreas en que por sus características puede ser de utilidad para los medios de comunicación. Así mismo exploran diversas experiencias de aplicación de blockchain al periodismo, Civil entre ellas. Según estos autores, las áreas en que blockchain puede ser aplicado al periodismo son seis:

- combatir las fake news y la desinformación;

- preservar la propiedad intelectual;

- limitar los sesgos y la influencia externa;

- resistir la censura;

- proteger a las fuentes que proporcionan información sensible (whistleblowers);

- fomentar la creación de contenidos por parte de los usuarios.

Algunas de las alternativas que la tecnología blockchain ofrece al periodismo pueden leerse en el monográfico Blockchain y periodismo (Marqués-Pascual; Sintes-Olivella, 2020).

Covadonga Fernández asegura que, en un entorno como el actual, en que abundan la desinformación y las fake news,

"con la blockchain puede conocerse el origen de las noticias que, después, se constatan falsas. Un origen que, en la medida de la defensa del anonimato que permite la propia blockchain, puede ser anónimo" (Fernández, 2017, p. 88).

En este mismo sentido, Don y Alex Tapscott están convencidos de que

"Ios gobiernos no podrían destruir o alterar la información registrada en la blockchain, por lo que podríamos usarla para pedirles responsabilidades a ellos y a otras instituciones" (Tapscott; Tapscott, 2017, p. 350).

En el ámbito económico, la criptomoneda -los medios pueden crear su propia criptomoneda o usar alguna ya existentepermite a los usuarios

"comprar, por fracciones de euro o bitcoins, artículos, reproducciones de vídeos, noticias, fotos, dibujos, suscripciones al medio por páginas o secciones concretas, minutos de lectura, horas y días" (Fernández, 2017, p. 85).

La experiencia pionera más relevante en periodismo ha sido Civil (https://civil.co), una plataforma que proveía tecnología blockchain a medios de comunicación. Raymond Colle explica en su libro Blockchain para periodistas y medios de comunicación que

“en agosto 2017, pude encontrar ya algunas organizaciones que presentaban proyectos de plataformas periodísticas operativas en blockchain como Civil, DNN, Steemit y Deecent. Excepto DNN, se trata de empresas que no son medios de comunicación, sino que ofrecen una plataforma para periodistas" (Colle, 2017, p. 18).

Hemos considerado oportuno dedicar nuestra atención a Civil, no sólo por su condición de proyecto pionero, sino también por el número de publicaciones que decidieron sumarse a este proyecto estadounidense, que desafortunadamente desapareció en junio de 2020.

\section{Objetivos y método}

Utilizando las metodologías asociadas al estudio de caso (Wimmer; Dominick, 1996), el objetivo general de este artículo es analizar el caso de Civil, la primera plataforma blockchain para periodismo. 
Como objetivos específicos, se propone:

- explicar la misión y los objetivos del proyecto;

- contextualizar el origen y la historia de Civil;

- determinar la estructura de la organización;

- detallar cómo diseñó su gobernanza y conocer su modelo de organización, explorando la función de la criptomoneda propia (CVL);

- analizar sus principios y normas profesionales y éticas rectores.

En primer lugar, se llevó a cabo un estudio del proyecto a partir de la información publicada en el White paper de la organización, así como en su web y el blog de Civil, que incluían publicaciones relevantes sobre el origen, características y evolución del proyecto. En paralelo, se realizó una extensa revisión bibliográfica -incluidos los numerosos artículos aparecidos en prensa- sobre periodismo y blockchain, así como, concretamente, sobre el proyecto Civil.

Posteriormente, se estableció contacto y se realizaron entrevistas a varios responsables de la organización en dos fases. El 8 de febrero de 2019, Matt Coolidge, cofundador y responsable de marketing de Civil de septiembre de 2017 a marzo de 2019, y en el momento de realizarse la entrevista consejero de Civil, respondió a un cuestionario elaborado por los autores de este artículo. En esta primera fase de la investigación se realizaron dos entrevistas exploratorias a Úrsula O’Kuinghttons, líder regional para América Latina y Europa de Civil Media-como se explica más adelante, Civil se dividió en dos organizaciones, Civil Media y la Fundación Civil-, el 27 de junio de 2018 y el 8 de febrero de 2019. Al mismo tiempo, el 4 de enero de 2019, se entrevistó a María Bustillos, editora del periódico digital Popula en Civil, con sede en Los Ángeles (California), la primera redacción que archivó un artículo en la plataforma norteamericana, cuya tecnología blockchain está basada en el sistema creado por Ethereum.

Así pues, además del cuestionario contestado por Coolidge, se llevaron a cabo tres entrevistas exploratorias para conocer de primera mano el modelo de organización de Civil y abrir la puerta a nuevas consideraciones planteadas por los entrevistados. Estas primeras entrevistas exploratorias permitieron obtener datos relevantes para comprender el proyecto de Civil.

Posteriormente, en junio de 2019, se realizó una visita a las oficinas de Civil en Nueva York. Fruto de esta visita, resultaron las entrevistas en profundidad mantenidas con el CEO y fundador de Civil, Matthew Iles; Liv Buli, vicepresidenta del equipo editorial de Civil Media hasta unos meses antes de la redacción de este artículo, y Nicole Bode, cofundadora y responsable de estrategia del primer conjunto de redacciones de Civil, que dejó la organización tras el verano de 2019. En las entrevistas se trataron asuntos como la historia del lanzamiento y del desarrollo del proyecto, el modelo de organización y de negocio de la plataforma, su sistema de gobernanza y la función de la criptomoneda propia (CVL token), el proceso de selección e incorporación de redacciones, y la política de ética periodística que regía su actividad. Las entrevistas en profundidad realizadas en la sede de Civil en Nueva York permitieron a los investigadores ahondar en el conocimiento de la organización. Para la realización tanto de las entrevistas exploratorias como de las entrevistas en profundidad se adoptaron los planteamientos teóricos de Quivy y Carnpenhoudt (1997).

Finalmente, durante la elaboración de este artículo, se llevaron a cabo diversas consultas mediante correo electrónico a Vivian Schiller, jefa del Consejo de la Fundación Civil, y antigua presidenta y CEO de la National Public Radio de EUA y ex vicepresidenta senior y responsable digital de NBC News.

\section{Resultados}

\subsection{Misión y objetivos de Civil}

Civil se definía a sí misma, en la introducción de su Constitución (Civil, 2018a), como una red comunitaria que "busca promover un periodismo confiable y sostenible", y subrayaba el principio de que una prensa libre es esencial para una sociedad justa y equitativa. Se añadía que Civil estaba construida usando la tecnología blockchain, algo que permite el almacenamiento de contenidos, la gestión y gobernanza de la red a cargo de sus participantes y

"un sistema de pago confiable para que el público financie, encargue, consuma y contribuya al periodismo".

Más adelante en el mismo documento, al referirse al "propósito de Civil", se es algo más específico. Tras afirmar que Civil se dirigía primero y ante todo al público, al que el periodismo está destinado a servir, se resaltaba que su finalidad era la de proporcionar a los ciudadanos información que "les permita participar plenamente en la sociedad". A este fin, Civil buscaba establecer las condiciones para que el periodismo pudiera cumplir este propósito:

"con mínima interferencia del gobierno, presiones comerciales, u otros intereses que intentan inadecuadamente influenciar, controlar o detener la recolección y difusión de hechos, opiniones e ideas en la esfera pública a través de leyes injustas, presión comercial, intimidación o violencia" (Civil, 2018a).

María Bustillos señalaba en este sentido que

"Popula ya ha logrado demostrar el valor de archivo de blockchain para el periodismo al crear archivos más allá del alcance de censores o gobiernos hostiles y opositores a la libertad de prensa".

En palabras de Liv Bulli, vicepresidenta del equipo editorial de Civil Media, 
"Civil es una plataforma comunitaria para el periodismo independiente".

En el White paper de Civil, redactado por el fundador y CEO Matthew Iles con anterioridad a la Constitución, se definía Civil como un protocolo de comunicaciones descentralizadas para periodistas y ciudadanos. Se insistía asimismo en las ventajas de no tener que depender de terceras partes. En la introducción del mismo documento, lles proclamaba que existe una

"tremenda oportunidad para un nuevo y sostenible modelo

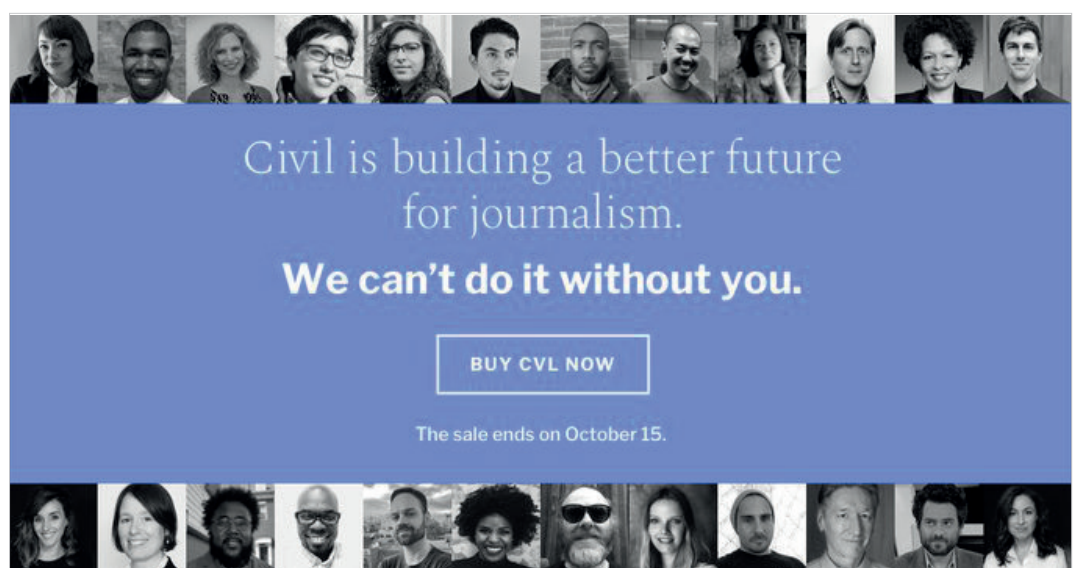

Figura 2. Tweet invitando a comprar Civil tokens (CVLs)

https://twitter.com/Sludge/status/1051625003781902341 para los periodistas" ya que el modelo actual "no premia el servicio a sus comunidades por encima de todo lo demás" (Civil, 2018b).

En la entrevista realizada en Nueva York, lles apuntó que el grupo fundador quería contribuir a un determinado tipo de periodismo, y que de ahí nació la idea de elaborar una Constitución. Para él,

"el modelo de negocio de las redacciones se basa en el público. No en la publicidad, a pesar de que algunas tienen publicidad".

Y definía cuál es su visión de lo que el periodismo debe ser:

"Veo el periodismo como la manera a través de la que la gente se informa sobre su mundo y toma decisiones en ese contexto. Pienso que el mayor objetivo del periodismo es este: ayudar a la sociedad a estar informada, conectada e implicada [engaged]".

Aspiraba asimismo a que Civil se convirtiera en una voz relevante en EUA:

“Dentro de un año -la entrevista se realizó en junio de 2019- queremos que una masa crítica de personas se informe a través de Civil y que reconozca la diferencia entre cómo Civil gestiona los contenidos en contraposición a Facebook y Google".

En un post de agosto de 2018, lles había vaticinado que la "nueva economía para el periodismo" será "autogobernada", es decir, que

"Ios individuos deciden cómo proteger y evolucionar el sistema con el tiempo, no los gigantes tecnológicos, los intereses especiales o los gobiernos" (lles, 2018).

Por su parte, el consejero de Civil Matt Coolidge, entrevistado en la primera fase de este estudio, exponía que:

"Civil está experimentando con un modelo radicalmente nuevo, en el que hemos construido una plataforma y, posteriormente, le estamos entregando el control a una comunidad de miembros que se preocupan por apoyar el periodismo de calidad y están experimentando con nuevas formas de apoyar directamente a los periodistas, mientras que al mismo tiempo les permiten capturar una mayor parte del valor que crean. Esto es posible gracias a la naturaleza descentralizada (propiedad de la comunidad) de Civil, donde ninguna de las partes tiene el control, y todos están incentivados para cooperar".

\subsection{Origen e historia de Civil}

Civil nació oficialmente en noviembre de 2016. Su principal impulsor fue Matthew lles, CEO hasta el final de Civil. Tras graduarse por la Duke University (Carolina del Norte) en 2009, fundó dos compañías y trabajó en una tercera, todas ellas relacionadas con el marketing digital antes de emprender el proyecto Civil.

En 2016 empezó a reflexionar sobre la idea de poner en marcha una nueva start-up. Esta vez tenía claro que no estaría destinada a ayudar a la comercialización de productos o servicios de otras empresas. En lugar de ello, deseaba focalizarse en el mundo del periodismo y la distribución de contenidos. Como él recordaba al ser entrevistado, en aquellos momentos Facebook y Google ya eran muy poderosos. Admiraba y se había interesado a fondo por conocer y comprender "el modelo de Wikipedia". Igualmente, en la entrevista subrayó la inspiración que supuso para él el profesor y ensayista Clay Shirky. Cuando descubrió blockchain se dio cuenta de que aquello podría ser su "arma Civil buscaba establecer las condiciones para que el periodismo pudiera cumplir este propósito: "mínima interferencia del gobierno, presiones comerciales, u otros intereses que intentan inadecuadamente influenciar, controlar o detener" secreta". 
En otoño de 2016, las bases de lo que debía ser el proyecto estaban listas. Iles las plasma en la publicación digital Medium (Iles, 2016). El empresario canadiense Joseph Lubin, cofundador de Ethereum y fundador de ConsenSys, compañía dedicada a soluciones informáticas basadas en la tecnología blockchain, decide apostar por el proyecto e invertir 5 miIlones de dólares en él (Hazard-Owen, 2018). Así nació Civil Media, empresa convencional dedicada a servicios digitales y de blockchain, que posibilitó la creación de la parte not-for-profit de Civil, la Fundación Civil.

Civil se puso realmente en marcha en 2017. El primer borrador de su Constitución se publicó en mayo de 2018 a partir de las aportaciones de académicos, periodistas y del público. Las primeras redacciones adscritas fueron catorce, que recibieron una fuerte ayuda financiera de Civil. La segunda versión de la Constitución fue publicada tres meses después, en agosto. Como se ha comentado ya, la plataforma blockchain sobre la que operaba Civil se basaba en el sistema Ethereum. La mayoría del equipo fundacional siguió ocupando posiciones de responsabilidad durante buena parte del trayecto. Sus perfiles profesionales eran tanto periodísticos como tecnológicos.

En agosto de 2019, Civil contaba con 59 redacciones admitidas en el Registro de Civil, es decir, que pasaban a estar integradas en la plataforma, mientras que en febrero de 2020 había llegado a 75. Estas cifras quedaban lejos de los objetivos inicialmente planteados por Civil. La meta era tener un millar de redacciones asociadas a la plataforma en verano de 2020 pero, como se verá más adelante, a principios de junio de 2020 Civil su cierre.

\subsection{Estructura de la organización}

Civil, como se ha apuntado más arriba, se articulaba en dos grandes áreas. La primera era Civil Media, en la que trabajaban unas quince personas y que era fundamentalmente una compañía tecnológica; y la segunda era la Fundación Civil, puramente periodística, sin ánimo de lucro y con alrededor de media docena de empleados.

La Fundación acogía el llamado Consejo de Civil, formado por profesores y periodistas de prestigio a nivel internacional. El Consejo era la instancia de apelación de Civil. Al frente del Consejo se encontraba la ya citada Vivian Schiller, con una larga carrera a sus espaldas como ejecutiva de medios. Del Consejo formaba parte el español Javier Moreno, director de El país desde el pasado junio y quien ya había estado al frente del rotativo en una etapa anterior..

"El Council vela para que el contenido de la Constitución de Civil se efectúe y se maneje correctamente dentro de las propias redacciones",

recalcó Ursula O’Kuighttons, líder regional para América Latina y Europa de Civil Media, en la entrevista realizada para este estudio.

La Comunidad Civil vendría a ser la asamblea o parlamento. Estaba compuesta por las redacciones miembros de Civil y por todos aquellas entidades o ciudadanos que poseían Civil tokens (CVL), la criptomoneda creada por Civil. En cuanto a las redacciones que integraban la Comunidad Civil, como explicó Coolidge,

"existe un amplio conjunto de redacciones en Civil, pero la mayoría se enmarca en uno de estos dos segmentos: periodismo local o de investigación".

"Algunas redacciones buscan obtener beneficios, son for-profit, otras son non-profit",

comentó Nicole Bode, cofundadora y responsable de la estrategia del primer conjunto de redacciones que se integraron en Civil. Así pues, "algunos [medios] han puesto paywall, o algún tipo de pago, algunos piden donaciones, otros tienen suscripciones, etcétera".

Civil mantenía a su vez alianzas con Forbes, la agencia Associated Press y WordPress, entre otras compañías.

\subsection{Modelo de organización, gobernanza y función de la criptomoneda}

La principal función del Consejo era resolver las apelaciones de la Comunidad sobre denuncias o desafíos (challenges) contra una redacción candidata al ingreso en Civil o bien contra una redacción que ya era miembro. Estas denuncias o desafíos se sometían a discusión en el seno de la propia Comunidad, que analizaba si la labor de una determinada redacción se ajustaba a la Constitución de Civil o si su forma de proceder la contravenía. La Comunidad aprobaba o rechazaba las candidaturas de las nuevas redacciones y podía llegar a ordenar la expulsión de una redacción que era ya miembro de la plataforma.

Tanto los challenges como el voto estaban vinculados a un sistema de incentivos positivos y negativos (con recompensas y castigos mediante $C V L S$ ) para estimular tanto la participación como la responsabilidad.

El Consejo actuaba como instancia de apelación y podía revertir las decisiones de la Comunidad. A su vez, la Comunidad poseía la capacidad última de vetar una resolución del Consejo, siempre que se alcanzara una mayoría del $66,7 \%$ de los votos (ponderados según la cantidad de CVLs en poder de cada miembro) (Civil, 2018c). Se produjeron diversos de estos challenges y también algunos rechazos y expulsiones.
La Comunidad Civil aprobaba o rechazaba las candidaturas y podía ordenar la expulsión de una redacción que formara parte de la plataforma 
En la Constitución de Civil se especificaba como objetivo para el año 2020 el autogobierno (self-management) por parte de la Comunidad (Civil, 2018c). Como destacó Iles en las manifestaciones recogidas para este artículo,
La posesión de los tokens de Civil (CVLs)

otorgaba el derecho a formar parte y participar en la llamada Comunidad Civil

"blockchain no es sólo tecnología. Hay también

una forma de gobernanza facilitada por blockchain, trabaja a través de la gente, hay una especie de contrato social".

Desde su inicio, Civil quiso poder tener una criptomoneda propia, con dos funciones principales:

- facilitar las interrelaciones económicas entre plataforma, redacciones y público, cosa nunca fue implementada;

- forjar una comunidad sólida en torno a la plataforma y sus medios asociados.

En septiembre de 2018 Civil lanzó una Initial Coin Offering (ICO) de su criptomoneda, CVL. Su objetivo era vender CVLS por valor de entre 8 y 24 millones de dólares. No obstante, la oferta fracasó pues se compró moneda por poco más de 1,4 millones.

Pese a que Civil había despertado ya por entonces un notable interés entre importantes medios de comunicación estadounidenses, el éxito no se trasladó a su criptomoneda. Una de las causas que se apuntan del fracaso es la complejidad del proceso de adquisición de CVLs, que requería un total de 44 pasos. Parece corroborar este argumento el hecho de que 1.738 compradores acabaron abandonando el proceso de compra tras registrarse (los que completaron todo el proceso fueron solo 1.012) (Clark, 2018).

Civil volvió a lanzar su venta de tokens en marzo de 2019, empleando un proceso mucho más fácil y simple. Esta vez fue mejor y la Comunidad Civil funcionó de este modo hasta el día del cierre.

\subsection{Principios, normas profesionales y éticas rectores}

En el apartado de "Política de ética periodística", de la Constitución de Civil, se dejaba claro que los principios especificados en ella no pretendían reemplazar o imponerse a las pautas éticas de cada redacción. Lo que se perseguía en primer lugar, se resaltaba, es un periodismo "independiente, basado en los hechos y responsable". Por su parte, Liv Buli enfatizó que

"todas las redacciones aprobadas en el Registro Civil se han comprometido públicamente con los principios establecidos en la Constitución de Civil. Además de esto, la mayoría, si no todas las redacciones, tienen su política editorial".

Los principios que se citan en la Constitución son trece, algunos de los cuales comentamos:

- Exactitud, en que se incluye la verificación, la consulta de fuentes directas y también indicar de dónde proviene la información que se ofrece;

- Presentar los hechos desde diferentes puntos de vista;

- Transparencia, que obliga a las redacciones a, entre otras cuestiones, a revelar sus fuentes de financiación e informar de posibles conflictos de intereses;

- Señalar claramente la diferencia entre contenidos editoriales y contenidos publicitarios u otros contenidos patrocinados;

- Esforzarse en responder a las críticas y otros comentarios del público;

- Adoptar mecanismos de autorregulación interna, como el nombramiento de un editor de lectores o un defensor del lector.

Es pertinente por su interés reproducir literalmente el octavo punto, que apela a los periodistas a evaluar y ser conscientes de las consecuencias para terceros de la difusión de determinadas informaciones. Específicamente dice así:

“Respeto y consideración al impacto que tiene el periodismo en las vidas de los otros. Esto incluye equilibrar la necesidad del público de ser informado contra cualquier daño potencial, y mostrar compasión por quienes pudiesen verse afectados por la cobertura de las noticias" (Civil, 2018a).

\section{Conclusiones}

Civil fue una plataforma de redacciones que tenía como misión el impulso de un periodismo independiente, veraz y de calidad. El proyecto se basaba en la firme aplicación de los principios éticos y profesionales del periodismo y en sacar provecho de las ventajas propias de la tecnología blockchain.

Civil empezó su andadura gracias a la financiación de la compañía ConsenSys, propiedad del canadiense Joseph Lubin, cofundador asimismo de Ethereum. Civil dependió siempre de la financiación de ConsenSys. La organización, no obstante, se propuso desde el principio conseguir que esa dependencia financiera fuera menguando gracias a la incorporación de nuevas fuentes de financiación que aseguraran la sostenibilidad de la plataforma a largo plazo, algo que no consiguió.

Civil se estructuraba en dos grandes entidades, Civil Media y la Fundación Civil, que era la parte periodística y sin ánimo de lucro. La Fundación contaba con el Consejo de
Ganar la mayor masa crítica posible era transcendental para el éxito del proyecto 
Civil, formado por profesores y periodistas de prestigio. El Consejo actuaba como la instancia de apelación de la Comunidad Civil -formada por todos aquellos que poseían CVLs, la criptomoneda creada por Civil. Al frente del Consejo se encontraba Vivian Schiller.

La criptomoneda de Civil se usaba sobre todo para la gobernanza de la plataforma, pues, como se ha indicado, la posesión de los tokens de Civil (CVLS) era lo que otorgaba el derecho a formar parte y participar en la llamada Comunidad Civil. El voto se ponderaba en relación con la cantidad de CVLs de cada miembro. Es por ese motivo que los CVLS podían compararse con las acciones o participaciones en una sociedad mercantil.

El sistema de autorregulación de Civil se fundamentaba en la capacidad de cualquier miembro de la comunidad para lanzar denuncias o desafíos (challenges) contra aquellas redacciones candidatas que no se considerasen adecuadas para ingresar en la organización. Asimismo, podía hacerlo también contra redacciones que ya formaran parte de Civil si se estimaba que estaban desatendiendo los principios fijados en la Constitución. Tales challenges se sometían a debate y votación en el seno de la Comunidad. Ésta podía, tras este proceso, negar el ingreso a una redacción o incluso expulsar a una redacción ya miembro.

La Constitución Civil, con la cual todo miembro debía comprometerse explícitamente, fijaba los principios éticos que los medios o redacciones tenían que respetar. El texto establecía trece principios. El primero de ellos recalca el valor de la "exactitud", en que se incluye la verificación, la consulta de fuentes en primera persona e informar sobre de dónde proviene la información.

Por una parte, Civil imponía un fuerte control sobre la calidad de los contenidos, control que -como se ha mencionado- tenía su normativa de referencia en la Constitución. Por otra, concedía libertad a los diferentes medios en cuanto a su modelo de negocio concreto. De este modo, por ejemplo, convivían redacciones con ingresos publicitarios con otros que no contaban con ellos.

A principios de junio de 2020 se supo públicamente que Civil iba a cerrar. El principal motivo de la decisión fueron los problemas financieros (Edmonds, 2020). La compañía que había propiciado la creación de Civil y que la había ido financiando desde entonces, ConsenSys, venía arrastrando problemas desde finales de 2018. Una vez decidido el cierre de Civil, sus trabajadores se incorporaron a Consensys.

Civil, que financió generosamente a los primeros medios que se incorporaron a la plataforma, realizó un gran esfuerzo por acelerar la incorporación de nuevas redacciones. Ganar la mayor masa crítica posible era indispensable. Sin embargo, la cantidad de afiliaciones efectivamente alcanzadas siempre quedó muy corta respecto a los ambiciosos objetivos establecidos.

\begin{abstract}
It's with a heavy heart that I announce the end of Civil. The Civil team and technology will be joining ConsenSys to build identity solutions on Ethereum.
\end{abstract}

In 2016, Civil was founded on a moonshot mission to create a blockchain-based media platform for trustworthy journalism owned and operated by the public. We set out to decentralize how the news is vetted, how journalism is funded, and how we stay informed as a society. We were among the first startups in the world to experiment with blockchain and cryptocurrencies in the media space. We built innovative technology, supported award-winning journalists, and inspired many people all over the world with our vision for a more participatory media landscape. But ultimately, we failed to sustain ourselves independently.

Several months ago, we started to develop products related to decentralized identity in the media and advertising space, which attracted enterprise interest for use cases such as trackable content licensing and transparent ad decisioning. This pivot led to closer coordination with ConsenSys and the team building solutions for identity and provenance tracking, which in turn started conversations about a strategic merger. We are excited to share that the Civil team and technology will join ConsenSys to be a part of these efforts. Although the journey for Civil is over, our new team continues to develop cutting-edge technology that I believe will contribute to building a better internet. This isn't the outcome we had envisioned, but nevertheless, we're proud of what we accomplished. We couldn't have done it without the support of our passionate community.

Newsrooms on Civil have always operated independently, and therefore will remain unaffected. The Civil Registry, Civil tokens, and other work are open-source and operational, but there will be no further active development or management on our part. The Civil Foundation's future is uncertain, but it is effectively in hibernation for now.

Civil will always hold a special place in my heart. Our grand experiment did not achieve its mission, but we did succeed in bringing many people together around something radically different and in service of something deeply important. Our mission for journalism is more important now than ever. I'm grateful for our chance to make a difference.

Thank you so much to all of our teammates, partners, newsrooms, and supporters. You made the journey unforgettable.

Sincerely,

Matthew Iles, Civil CEO
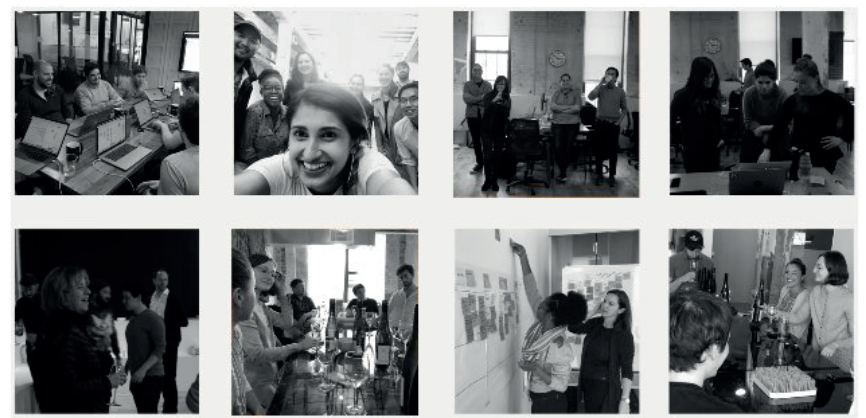

(C) 2020 The Civil Media Company

Figura 3. Ending of the Civil Journey

https://civil.co 
Civil proporcionaba la plataforma tecnológica y ofrecía servicios y herramientas de utilidad a las redacciones asociadas a fin de que pudieran mejorar el servicio a su público, crecer y convertirse en económicamente rentables. El proyecto dependía en parte del desarrollo y fortalecimiento de las salas de redacción que con el tiempo debían contribuir a financiar la plataforma. En esta línea, en agosto de 2019 Civil lanzó su propio sistema de crowdfunding, llamado Boosts.

Se ha venido criticando largamente, y se subrayó nuevamente en el momento en que lles confirmó el cierre de Civil (Iles, 2020), que uno de los puntos débiles del proyecto era su extrema complejidad tecnológica, por una parte, pero también, y quizá más relevante, de su sistema de gobernanza. Realmente el proyecto era extremadamente difícil de explicar de forma clara y sencilla. No deja de ser paradójico o un contraste que, mientras Civil se basaba en la tecnología de bloques -una de cuyas características definitorias es la descentralización- para que las redacciones publicaran sus contenidos, en cambio el sistema de gobernanza estuviera completamente centralizado. Así pues, eran los miembros de la Comunidad y del Consejo de Civil -en ningún caso el público o los ciudadanos- quienes ejercían la vigilancia sobre los contenidos que se publicaban a través de la plataforma.

Aún es temprano para señalar si la tecnología blockchain aplicada al periodismo será útil para recuperar la confianza del público y si permitirá aumentar progresivamente los ingresos económicos y dar estabilidad financiera a los medios que realicen esta apuesta. Es necesario sin duda un período más prolongado en el tiempo para que pueda apreciarse debidamente lo que puede aportar al periodismo una tecnología que se encuentra todavía en desarrollo. En especial en cuanto a, por una parte, su velocidad y, por otra, los costes asociados al sistema de procesamiento y verificación de los bloques de información.

En su carta anunciando el cierre de Civil, lles (2020) recuerda:

“Nuestro gran experimento no logró su misión, pero sí tuvimos éxito en reunir a muchas personas en torno a algo radicalmente diferente y al servicio de algo profundamente importante. Nuestra misión para el periodismo es más importante ahora que nunca. Estoy agradecido de haber tenido la oportunidad de cambiar las cosas".

Pese a su corto recorrido, el camino abierto por Civil es, en especial en cuanto a algunos de sus elementos, muy valioso y a su vez ciertamente esperanzador.

\section{Referencias}

Al-Saqaf, Walid; Picha-Edwardsson, Malin (2019). "Could blockchain save journalism?: An explorative study of blockchain's potential to make journalism a more sustainable business". In: Ragnedda, Massimo; Destefanis, Giuseppe (eds.) Blockchain and web 3.0. Londres: Routledge, pp. 97-113. ISBN: 9780429029530

Anderson, Chris W.; Bell, Emily J.; Shirky, Clay (2015). "Post-industrial journalism: adapting to the present". Geopolitics, history \& international relations, v. 7, n. 2, pp. 32-123.

https://doi.org/10.7916/D8N01JS7

Blumler, Jay G.; Kavanagh, Dennis (1999). "The third age of political communication: Influences and features". Political communication, v. 16, n. 3, pp. 209-230.

https://doi.org/10.1080/105846099198596

Boulosa, Nicolás (2019). Blockchain: ¿ffuego prometeico o aceite de serpiente? Madrid: Anaya. ISBN: 9788441541481

Civil (2018a). The Civil Constitution (versión en castellano). [Página no activa].

https://docs.google.com/document/d/1ZEdBgaZBRBNKUuRWBP7AuEVXyVa7uAFqJPG8pMg2keY/edit

Civil (2018b). Civil White Paper. [Página no activa].

https://civil.co/white-paper

Civil (2018c). The Civil Constitution. [Página no activa].

https://civil.co/constitution

Clark, Kate (2018). "Blockchain media startup Civil is issuing full records to all buyers of its cryptocurrency". TechCrunch, 17 October.

https://techcrunch.com/2018/10/16/blockchain-media-startup-civil-is-issuing-full-refunds-to-all-buyers-of-itscryptocurrency

Colle, Raymond (2017). Blockchain para periodistas y medios de comunicación. Santiago de Chile: Ediciones Incom-Chile. Asociación Chilena de Investigadores en Comunicación. http://incomchile.cl/wp-content/uploads/2012/03/Blockchain-periodismo.pdf

Edelman (2020). Edelman Trust barometer. Global report. Edelman.

https://edl.mn/2NOw/tm 
Edmonds, Rick (2020). "R.I.P. Civil-Lessons from a failed startup". Poynter, 2 June.

https://www.poynter.org/business-work/2020/r-i-p-civil-lessons-from-a-failed-startup

Fernández, Covadonga (2017). “Medios de comunicación y la blockchain”. En: Preukschat, Alexander (coord.). Blockchain: la revolución industrial de Internet. Barcelona: Gestión 2000, pp. 83-88. ISBN: 9788498754476

Friedman, Thomas L. (2018). Gracias por llegar tarde. Barcelona: Ediciones Deusto. ISBN: 9788423429097

Hazard-Owen, Laura (2018). "Civil's token sale has failed. Now what? Refunds, for one thing - and then another sale". Nieman Lab, 16 October.

https://www.niemanlab.org/2018/10/civils-token-sale-has-failed-now-what-refunds-for-one-thing

Iles, Matthew (2016). "The first post". Medium, 15 Nov.

https://medium.com/@matthewiles/the-first-post-fe4bf9fdb501

Iles, Matthew (2018). "Why join the Civil economy. Or, why buy CVL tokens”. Blog join Civil, 24 August. [Página no activa]. https://blog.joincivil.com/why-join-the-civil-economy-497070d5267f

Iles, Matthew (2020). "Ending of the Civil journey". Civil.

https://civil.co

Jarvis, Jeff (2014). Geeks bearing gifts: Imagining new futures for news. New York: CUNY Journalism Press. ISBN: 9781 939293732

Marqués-Pascual, Joaquín; Sintes-Olivella, Marçal (eds.) (2020). Blockchain y periodismo. Cómo la cadena de bloques cambiará a los media. Barcelona: Editorial UOC. ISBN: 9788491806028

Pew Research Center (2019). "Newspapers fact sheet". Pew Research Center. Journalism \& media, 9 July.

https://www.journalism.org/fact-sheet/newspapers

Quivy, Raymond; Van-Carnpenhoudt, Luc (1992). Manual de investigación en ciencias sociales. México, D.F.: Limusa. ISBN: 9789681843557

http://www.fapyd.unr.edu.ar/wp-content/uploads/2015/09/manual-de-investigacion-en-ciencias-sociales-quivycampenhoudt.pdf

Sunstein, Cass R. (2002). Republic.com. Princeton (USA) \& Oxford (UK): Princeton University Press. ISBN: 9780691095899

Tapscott, Don; Tapscott, Alex (2017). La revolución blockchain. Descubre cómo esta nueva tecnología transformará la economía global. Barcelona: Ediciones Deusto. ISBN: 9788423426553

Wimmer, Roger D.; Dominick, Joseph R. (1996). La investigación científica de los medios de comunicación. Barcelona: Bosch Comunicación. ISBN: 9788476763599.

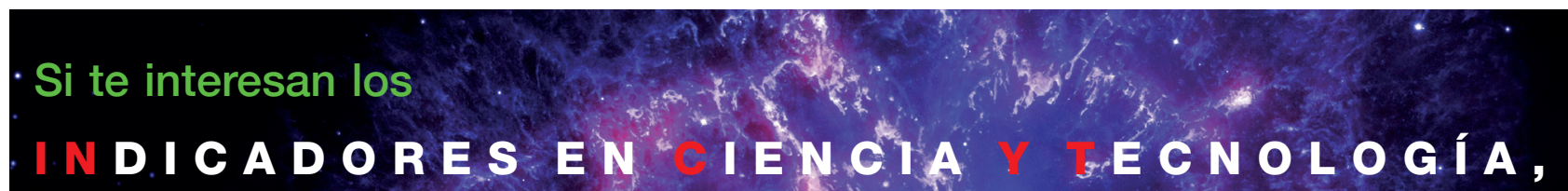

y todos los temas relaciona dos con litmédición de la ciểisia, tales como:

Análisis de citàs, Normalización dénompreš́e instituciones, Impacto de

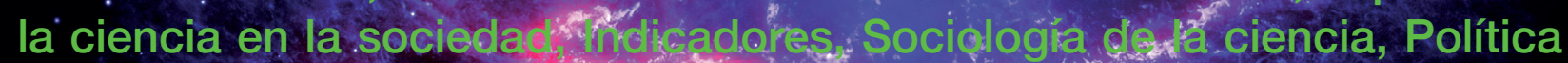
científica, Comunicación de la cien sia, Revistasnges de datos, Índices de impacto, Políticas de open accetss, Análisistid la nueva economía, Mujer y ciencia, etc.

\section{Éntonces es tu lista. Suscríbete en:}

http://whw.rediris.es/list/info/incyt.htmI 